\title{
Ambulatory percutaneous nephrolithotomy is safe and effective in patients with extended selection criteria
}

Gregory William Hosier; Kashif Visram; Thomas McGregor; Stephen Steele; Naji Touma; Darren Beiko

Department of Urology, Queen's University, Kingston, ON, Canada

Cite as: Hosier GW, Visram K, McGregor T, et al. Ambulatory percutaneous nephrolithotomy is safe and effective in patients with extended selection criteria. Can Urol Assoc J 2021 November 18; Epub ahead of print. http://dx.doi.org/10.5489/cuaj.7527

Published online November 18, 2021

Corresponding author: Dr. Gregory William Hosier, Department of Urology, Queen's University, Kingston, ON, Canada; gregory.hosier@gmail.com

$* * *$

\begin{abstract}
Introduction: Ambulatory percutaneous nephrolithotomy (PCNL) has been limited to highly selected patients. The objective of our study was to compare complication and stone-free rates after ambulatory PCNL in standard selection criteria vs. extended criteria patients.
\end{abstract}

Methods: We conducted a retrospective review of prospective data on all patients who underwent ambulatory PCNL at one academic center from 2007-2018. Extended criteria patients were defined as one or more of: age $>75$ years, body mass index (BMI) $>30 \mathrm{~kg} / \mathrm{m}^{2}$, American Society of Anesthesiologists (ASA) >2, bilateral stones, solitary kidney, transplant kidney, complete staghorn calculi, stone burden $>40 \mathrm{~mm}$, multiple tracts, or prior nephrostomy tubes/stents. Primary outcomes were complication rates (Clavien-Dindo classification) and stone-free rates.

Results: We identified 118 patients, of which 92 (78\%) met extended criteria. Mean BMI was 31 $\mathrm{kg} / \mathrm{m}^{2}$ and $45 \%$ were ASA 3 or higher. Mean sum maximum stone diameter was $24 \mathrm{~mm}$. Multiple stones were present in $25 \%$, bilateral stones in $7 \%$, and complete staghorn stones in $4 \%$ of patients. There was no difference in complication $(12 \%$ vs. $18 \%, \mathrm{p}=0.56)$, emergency department visit ( $12 \%$ vs. $18 \%, \mathrm{p}=0.56)$, or re-admission $(4 \%$ vs. $5 \%, \mathrm{p}=1)$ rates between standard and extended criteria patients respectively. Of the complications, $85 \%$ were ClavienDindo grade 1. Stone-free rates were not different between standard (84\%) and extended (83\%) criteria patients $(\mathrm{p}=1)$. No extended criteria variables were associated with complications in multivariable analysis. 
Conclusions: Complication and stone-free rates were not different between standard and extended selection criteria patients undergoing ambulatory PCNL. This data indicates that many of the preoperative patient and stone factors that have previously been used as exclusion criteria for ambulatory PCNL are not strictly necessary.

\section{Introduction}

Percutaneous nephrolithotomy (PCNL) is the standard minimally invasive surgical procedure for removal of kidney stones $>2 \mathrm{~cm}$. ${ }^{1}$ In traditional PCNL, patients are admitted to hospital for 1-3 days after surgery to monitor for post-operative bleeding, sepsis, or recurrent obstructive stones. Drainage of the kidney is typically achieved through a nephrostomy tube which provides access for a second procedure if necessary, and is then removed prior to discharge. Ambulatory PCNL has been described and offers potential benefits over inpatient PCNL including faster patient recovery, less pain related to the nephrostomy tube, and significant cost savings. ${ }^{2}$ In this technique, patients are discharged home the same day as surgery typically with a ureteric stent and no nephrostomy tube (tubeless). Multiple studies, including one randomized controlled trial, have shown that ambulatory PCNL is safe and effective in highly selected patients with minimal comorbidities and low stone burden..$^{2-10}$

A barrier to uptake of ambulatory PCNL has been use of highly selective pre-operative inclusion criteria, which precludes the majority of patients undergoing PCNL. In the only randomized controlled trial to date comparing ambulatory to inpatient PCNL, strict inclusion criteria including body mass index (BMI) $<30$, American Society of Anesthesiologists (ASA) class 1-2, no bilateral stones, total stone burden $<4 \mathrm{~cm}$, and single dilation tract were used. ${ }^{8}$ Similarly, stringent selection criteria have been used in most retrospective and prospective cohort studies published to date. ${ }^{4,5,7,10}$ This led authors of a recent systematic review to recommend the following exclusion criteria be used in patients undergoing ambulatory PCNL: ASA >2, BMI $>30$, multiple stones, renal anatomic abnormalities (including transplant kidney and solitary kidney), multiple tract dilations, or staghorn calculi. ${ }^{10}$ Consequently, the safety and efficacy of ambulatory PCNL in comorbid patients with large stone burden remains unknown.

Over our 10-year experience with ambulatory PCNL we began performing this technique in less highly selected patients. The objective of our study was to describe our experience with ambulatory PCNL to date and to compare complication and stone free rates in standard selection criteria vs. extended criteria patients from our cohort. 


\section{Methods}

\section{Study design}

This was a retrospective review of prospectively-collected data on all patients who underwent ambulatory PCNL at a single centre (Kingston Health Sciences Centre) from 2007-2018. Ontario-wide clinical and imaging data were queried. This study was approved by the Queen's University Health Sciences and Affiliated Hospitals Research Ethics Board.

\section{Patient selection criteria}

Initially, strict patient selection criteria were used as previously described. ${ }^{5,11}$ Briefly, this consisted of pre-operative exclusion criteria: ASA $>3, \mathrm{BMI}>35 \mathrm{~kg} / \mathrm{m}^{2}$, any renal anatomic abnormalities, bilateral stones, or pre-existing nephrostomy tube/ureteric stents. Intra-operative exclusion criteria were any: pelvicalyceal injury or significant bleeding intraoperatively. Postoperative exclusion criteria were any: fever, hemodynamic instability, or significant ongoing pain in the recovery room. After the initial 3-5 cases, these exclusion criteria were relaxed to only include: significant pelvicalyceal injury, significant intraoperative bleeding, hemodynamic instability, need for transfusion, fever, pneumothorax, hemothorax, or significant ongoing pain.

\section{Selection of variables for extended criteria}

Publications on ambulatory PCNL were reviewed for commonly utilized patient exclusion criteria. ${ }^{4,8-10}$ From these, we identified 10 commonly utilized exclusion criteria that were not used in our cohort. These were: age $>75$ years, BMI $>30 \mathrm{~kg} / \mathrm{m}^{2}$, ASA $>2$, bilateral stones, solitary kidney, complete staghorn calculi, transplant kidney, stone burden $>40 \mathrm{~mm}$, multiple tracts, or pre-existing nephrostomy tubes/stents.

\section{Operative procedure}

Operative technique did not change over the study period and has been published previously. ${ }^{5,11-}$

${ }^{13}$ Briefly, flexible cystoscopy was performed in the prone position and a 5 French ureteral catheter placed to perform retrograde pyelograms. A catheter was placed for bladder drainage. Fluoroscopic-guided needle puncture was performed using the eye of the needle technique. Balloon tract dilation and placement of a 30 French access sheath were performed. Rigid nephroscopy and lithotripsy were performed. Antegrade flexible nephroscopy, ureteroscopy, and basket extraction were performed as needed. A 6 French double-J stent was placed for kidney drainage in all but two patients (these were left totally tubeless). Patients were monitored in the recovery unit for 2-4 hours. Patients underwent a trial of void and were discharged home if they met discharge criteria: no hemodynamic instability (defined as 2 of 3: heart rate $>90$ beats per minute, respiratory rate $>20$ breaths per minute, systolic blood pressure $<90 \mathrm{mmHg}$, or drop in systolic blood pressure $>40 \mathrm{mmHg}$ ), no temperature $>38.5$ degrees Celsius, no hemoglobin drop of $>3 \mathrm{~g} / \mathrm{dL}$ compared to pre-operative bloodwork, no transfusion of blood products, no 
pneumothorax or hemothorax on chest radiography (CXR), no uncontrolled nausea/vomiting, and no significant pain. Indications for CXR were upper pole access or clinical concern.

\section{Primary outcomes}

Our primary outcomes were complication rate based on Clavien-Dindo classification and stone free rate defined as total residual stone burden $<3 \mathrm{~mm}$ as determined on imaging (ultrasound, computed tomography [CT], or kidney, ureter, bladder radiography [KUB]) 4-6 weeks after surgery.

\section{Statistical analysis}

Descriptive statistics were used for baseline characteristics and outcome data. Continuous baseline variables were checked for normality. A two-sided Student's t test was used for normally distributed continuous data. A Fischer's exact test was used for categorical data. Univariate and multivariable logistic regression analyses were performed. For multivariable analyses, all variables with $\mathrm{p}<0.2$ on univariate analysis were included. Assuming a complication rate of $15 \%$, we calculated that a sample size of 110 would be required to have $80 \%$ power to detect a $20 \%$ difference in rate of complications with a 2-sided alpha at level of 0.05. Data were analyzed using IBM SPSS Statistics for Mac, version 27 (IBM Corp., Armonk, NY, USA).

\section{Results}

We identified 118 patients of which $92(78 \%)$ met extended criteria. Mean BMI was $31 \mathrm{~kg} / \mathrm{m}^{2}$ and 45\% were ASA 3 or higher (Table 1). Mean sum maximum stone diameter was $24 \mathrm{~mm}$. Multiple stones were present in 25\%, bilateral stones in 7\%, complete staghorn stones in $4 \%$, and pre-existing nephrostomy tubes/stents in $4 \%$.

There was no difference in complication ( $12 \%$ vs. $18 \%, \mathrm{p}=0.56)$, Emergency department visit $(12 \%$ vs $18 \%, \mathrm{p}=0.56)$, or readmission $(4 \%$ vs. $5 \%, \mathrm{p}=1)$ rates between standard and extended criteria patients respectively (Table 2). Of the complications, $85 \%$ were Clavien-Dindo grade 1. No patients received blood transfusions. Stone free rates were not different between standard $(84 \%)$ and extended (83\%) criteria patients $(\mathrm{p}=1)$. Post-operative imaging modality was most commonly KUB (84\%) followed by CT (10\%) and US (6\%).

In univariate analysis, no extended criteria variables were associated with complications (Table 3). Stone burden $>40 \mathrm{~mm}$ (odds ratio [OR] 5.8, 95\% confidence interval [CI] 1.4-25.2, $\mathrm{p}=0.018$ ) and multiple tracts (OR 13.1, 95\% CI 1.1-154.7, $\mathrm{p}=0.041$ ) were associated with residual stone fragments in multivariable analysis (Table 4).

Two patients with extended selection criteria had high grade complications. One patient developed a non-ST elevation myocardial infarction six days after surgery. This patient had an uncomplicated surgery with normal post-operative hemoglobin. On presentation 6 days later, his hemoglobin remained normal with no signs of bleeding or infection. He was admitted for medical management and eventually underwent coronary artery bypass grafting for multivessel disease identified during his post-operative admission. 
The second patient with a high-grade complication underwent an uncomplicated left sided PCNL with an upper pole access (above $12^{\text {th }}$ rib) and was discharged after a negative CXR. He presented on post-operative day 1 to a peripheral hospital with left pleuritic chest pain and normal respiratory exam including normal oxygen saturation levels. He was given opioid pain medication and transferred to the tertiary care hospital (Kingston Health Sciences Centre). Upon assessment at the tertiary care hospital, he had signs of significant opioid toxicity including low respiratory rate, which required intubation. He underwent a CT scan which showed a moderate left pleural effusion and atelectasis with fluid appearance most consistent with hydrothorax. A chest tube was placed. The following day he was extubated and his chest tube removed after confirming resolution of the hydrothorax. He was discharged on post-operative day 4 in good condition.

\section{Discussion}

A number of retrospective and prospective studies report safety and efficacy of ambulatory PCNL, however studies to date have been limited to patients with minimal comorbidities and low stone burden, which precludes the majority of patients who undergo PCNL. Here we report our experience with ambulatory PCNL in patients using less stringent selection criteria. We found no difference in complication and stone free rates between standard and extended selection criteria patients undergoing ambulatory PCNL. In univariate and multivariable analyses, no patient or stone characteristics predicted for complications. This data indicates that many of the pre-operative patient and stone factors that have previously been used as exclusion criteria for ambulatory PCNL are not strictly necessary. Instead, we argue for a larger focus to be placed on the operative and immediate post-operative course when considering patients for same-day discharge.

Our overall complication rate of $17 \%$ and readmission rate of $5 \%$ is comparable to previous publications for PCNL. ${ }^{14}$ Our rate of high-grade complications (1.7\%) compares favourably with rates from the Clinical Research Office of the Endourological Society Percutaneous Nephrolithotomy Global Study, which reported 4\% grade 3-5 complications. ${ }^{14}$ Two recent systematic reviews of ambulatory PCNL trials reported complication rates of $13 \%$ with readmission rates of 3\%.9,10 All studies included in these reviews except one used strict selection criteria. From this review, authors recommended that the following exclusion criteria be used in patients undergoing ambulatory PCNL: ASA >2, BMI >30, multiple stones, renal anatomic abnormalities (including transplant kidney and solitary kidney), multiple tract dilations, or staghorn calculi. In contrast, our findings showed that no individual selection criteria were associated with increased odds of complications. Furthermore, patients with multiple extended selection criteria did not have higher rates of complications. This was using 30 French tracts, which would theoretically be higher risk for complications compared to smaller tracts. Similar to our findings, a retrospective cohort study by Bechis et al. found an acceptable complication rate of $20 \%$ with no Clavien-Dindo grade 3 or higher complications in a cohort that included $44 \%$ of 
patients with ASA 3 or higher, $20 \%$ with renal anomalies, and $17 \%$ with multiple tracts. ${ }^{6}$ Together, these data indicate that it is safe to consider ambulatory PCNL in less highly selected patients.

We found acceptable stone free rates in both standard and extended selection criteria patients at $84 \%$ and $83 \%$ respectively defined as total residual stone burden $<3 \mathrm{~mm}$ on KUB, ultrasound or CT scan at 4-6 weeks. These stone free rates are comparable to other published results. ${ }^{4,6,9,10}$ Stone burden $>4 \mathrm{~cm}$ and multiple tract dilations were identified as independent predictors of residual stones on multivariable analysis. These factors have been previously identified as predicting stone free rates and are variables in most predictive nomograms used for PCNL. ${ }^{15}$ Importantly, increased residual stones in our study did not lead to increased emergency department visits, readmissions, or additional procedures. In the only randomized controlled trial to date of ambulatory PCNL vs. inpatient PCNL, total stone burden $>4 \mathrm{~cm}$ was used as exclusion criteria. ${ }^{8}$ Based on a recent systematic review, authors recommended excluding patients with staghorn calculi or multiple punctures from consideration for ambulatory PCNL. ${ }^{10}$ However, our results showing no increased emergency department visits, readmissions, or need for secondary procedures in those with complex stones suggest that is reasonable to consider ambulatory PCNL in these patients.

The percentage of patients who underwent ambulatory PCNL rose from approximately $43 \%$ in 2013 to $74 \%$ in 2018. Although we did not specifically examine reasons for conversion of planned ambulatory surgery to inpatient surgery, Bechis et al found that post-operative nausea/vomiting $(41 \%)$ and social reasons (ie: no ride home, no one to stay overnight with patient; $35 \%$ ) were by far the most common reasons for conversion of ambulatory to inpatient PCNL. In our experience, social reasons are a very common reason for unplanned conversion of ambulatory to inpatient PCNL. This highlights the importance of clear communication with patients and all members of the care team about disposition planning starting from the preoperative visit.

An important potential downside of ambulatory PCNL is the risk of missing or delaying identification of serious complications that would otherwise be identified during a brief hospital admission after surgery. Two patients with extended selection criteria developed high grade complications. One patient developed a non-ST elevation myocardial infarction six days after surgery. Although it is possible that peri-operative hypotension played a role in development of his non-ST elevation myocardial infarction through demand-ischemia, the delayed timing of presentation (post-operative day 6) makes this somewhat unlikely. The delayed presentation also makes it unlikely that admission after PCNL would have resulted in this complication being identified earlier.

The second patient underwent an uncomplicated ambulatory PCNL with an upper pole access (above $12^{\text {th }}$ rib) and was discharged after a negative CXR. He presented with pleuritic chest pain and otherwise normal respiratory status initially, but developed respiratory failure 
after opioid administration that required intubation. A subsequent CT scan identified a moderate pleural effusion consistent with hydrothorax that was drained with a chest tube. It is unclear to what degree the hydrothorax contributed to the overall respiratory compromise given the normal respiratory exam on presentation to the peripheral hospital, the relatively small size of the hydrothorax on CT scan, and the co-occurrence of symptoms of significant opioid toxicity. In one study in which both routine CXR and CT chest were performed after PCNL in 100 patients, CXR identified hydropneumothorax in $8 \%$ but subsequent CT identified hydropneumothorax in $38 \%$ of patients. ${ }^{16}$ Of the $38 \%$ with hydropneumothorax on $\mathrm{CT}$ scan, only $7 \%$ required intervention of which only one was identified based on CT findings alone. ${ }^{16}$ Consequently, the vast majority of pulmonary complications with a negative CXR are not clinically significant in the absence of signs of respiratory compromise.

There are several potential benefits to ambulatory PCNL over inpatient PCNL. In the only randomized controlled trial to date, ambulatory compared to inpatient PCNL was associated with decreased hospital stay ( 0.5 versus 4 days), and fewer days to resume normal activities ( 8 versus 18 days). ${ }^{8}$ In a Canadian study, ambulatory PCNL was estimated to result in 35\% reduction in cost compared to inpatient PCNL. ${ }^{2}$ This worked out to a savings of $\$ 3000$ per PCNL. To date, most studies on ambulatory PCNL have focused on safety and efficacy without examining quality of life outcomes. Extrapolating from inpatient studies, tubeless PCNL is associated with improved post-operative pain scores and less analgesic use compared to PCNL with a nephrostomy tube in most studies, which suggests that ambulatory tubeless PCNL may have these same advantages. ${ }^{3,17}$ The importance of shifting elective procedures to the ambulatory setting whenever possible has been further highlighted during the COVID-19 pandemic.

Limitations of this study include potential selection bias given the non-randomized nature of the study. Although data was collected prospectively, the analysis was performed retrospectively which may have introduced bias. Since our study design only examined outcomes for patients discharged home the same day of surgery (and not outcomes for those that required post-operative admission), we were not able to assess reasons for conversion from planned ambulatory PCNL to inpatient PCNL or the overall safety and efficacy of PCNL at our center in those with extended selection criteria. However, the impact of patient comorbidities and stone burden on outcomes after inpatient PCNL have been examined extensively in other studies and was not the focus of our study. ${ }^{14,18-20}$ We also did not evaluate any patient reported outcomes. Strengths of our study were the large sample size (second largest ambulatory PCNL cohort that has been published). Another strength was that complete imaging and follow-up records were obtained in $97 \%$ of patients by analyzing all records captured in Ontario.

\section{Conclusions}

We found no difference in complication and stone free rates between standard and extended selection criteria patients after ambulatory PCNL. In univariate and multivariable analyses, no patient or stone characteristics predicted for complications. This data indicates that many of the 
pre-operative patient and stone factors that have previously been used as exclusion criteria for ambulatory PCNL are not strictly necessary. Instead, we argue for a larger focus to be placed on the operative and immediate post-operative course when considering patients for same-day discharge. 


\section{References}

1. Assimos D, Krambeck A, Miller NL, et al. Surgical Management of Stones: American Urological Association/Endourological Society Guideline, PART I. J Urol 2016;196:1153-60.

2. Kroczak T, Pace KT, Andonian S, et al. Ambulatory percutaneous nephrolithotomy in Canada: A cost-reducing innovation. Can Urol Assoc J 2018;12:427-9.

3. Chen ZJ, Yan YJ, Zhou JJ. Comparison of tubeless percutaneous nephrolithotomy and standard percutaneous nephrolithotomy for kidney stones: A meta-analysis of randomized trials. Asian Journal of Surgery 2020;43:60-8.

4. Schoenfeld D, Zhou T, Stern JM. Outcomes for Patients Undergoing Ambulatory Percutaneous Nephrolithotomy. J Endourol 2019;33(3):189-93.

5. Beiko D, Elkoushy MA, Kokorovic A, et al. Ambulatory percutaneous nephrolithotomy: What is the rate of readmission? J Endourol 2015;29:410-4.

6. Bechis SK, Han DS, Abbott JE, et al. Outpatient Percutaneous Nephrolithotomy: The UC San Diego Health Experience. J Endourol 2018;32:394-401.

7. Fahmy A, Rhashad H, Algebaly O, et al. Can percutaneous nephrolithotomy be performed as an outpatient procedure? Arab J Urol 2017;15:1-6.

8. Kumar S, Singh S, Singh P, et al. Day care PNL using 'Santosh-PGI hemostatic seal' versus standard PNL: A randomized controlled study. Cent Eur J Urol 2016;69:190-7.

9. Gao M, Zeng F, Zhu Z, et al. Day care surgery versus inpatient percutaneous nephrolithotomy: A systematic review and meta-analysis. Int J Surg 2020;81:132-9.

10. Jones P, Bennett G, Dosis A, et al. Safety and Efficacy of Day-case Percutaneous Nephrolithotomy: A Systematic Review from European Society of Uro-technology. Eur Urol Focus 2019;5:1127-34.

11. Beiko D, Lee L. Outpatient tubeless percutaneous nephrolithotomy: The initial case series. J Can Urol Assoc 2010;4:86-90.

12. Beiko D, Andonian S. Getting started with ambulatory PCNL. A CanMEDS perspective. J Can Urol Assoc 2015;9:223-5.

13. Ko R, Soucy F, Denstedt JD, et al. Percutaneous nephrolithotomy made easier: A practical guide, tips and tricks BJU Int; 2008;101:535-9.

14. De La Rosette J, Assimos D, Desai M, et al. The clinical research office of the endourological society percutaneous nephrolithotomy global study: Indications, complications, and outcomes in 5803 patients. J Endourol 2011;25(1):11-7.

15. Al Adl AM, Mohey A, Abdel Aal A, et al. Percutaneous Nephrolithotomy Outcomes Based on S.T.O.N.E., GUY, CROES, and S-ReSC Scoring Systems: The First Prospective Study. J Endourol 2020;34:1223-8.

16. Ogan K, Corwin TS, Smith T, et al. Sensitivity of chest fluoroscopy compared with chest $\mathrm{CT}$ and chest radiography for diagnosing hydropneumothorax in association with percutaneous nephrostolithotomy. Urology 2003;62:988-92.

17. Xun Y, Wang Q, Hu H, et al. Tubeless versus standard percutaneous nephrolithotomy: An update meta-analysis. BMC Urol 2017;17:1-17.

18. Desai M, De Lisa A, Turna B, Rioja J, et al. The clinical research office of the endourological society percutaneous nephrolithotomy global study: Staghorn versus 
nonstaghorn stones. Int Braz J Urol 2011;37:661.

19. Şahin A, Atsü N, Erdem E, et al. Percutaneous Nephrolithotomy in Patients Aged 60 Years or Older. J Endourol 2001;15:489-91.

20. Nouralizadeh A, Lashay A, Ziaee SAM, et al. Percutaneous Nephrolithotomy in HighRisk Patients: A Single-Center Experience with More than 350 Cases. Urol Int 2013;90:394-8. 


\section{Figures and Tables}

\begin{tabular}{|c|c|c|c|c|}
\hline Characteristic & $\begin{array}{c}\text { Overall, } \\
\text { n (\%) } \\
\text { N=118 }\end{array}$ & $\begin{array}{c}\text { Standard } \\
\text { criteria, } \\
\text { n }(\%) \\
\mathbf{N}=26\end{array}$ & $\begin{array}{c}\text { Extended } \\
\text { criteria, } \\
\text { n }(\%) \\
\mathrm{N}=92 \\
\end{array}$ & $\mathbf{p}$ \\
\hline Demographics & & 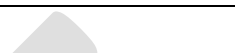 & & \\
\hline Age (years), mean (range) & $57(19-80)$ & $54(21-74)$ & $58(19-80)$ & 0.28 \\
\hline Female & $61(52)$ & $14(54)$ & $47(51)$ & 0.34 \\
\hline BMI $\left(\mathrm{kg} / \mathrm{m}^{2}\right)$, mean (range) & $31(19-82)$ & $26(19-29)$ & $32(20-82)$ & $<0.001$ \\
\hline \multicolumn{5}{|l|}{ ASA classification } \\
\hline 1 & $5(4)$ & $2(8)$ & $3(3)$ & 0.61 \\
\hline 2 & $58(50)$ & $24(92)$ & $34(37)$ & $<0.001$ \\
\hline 3 & $53(45)$ & 0 & $53(58)$ & $<0.001$ \\
\hline 4 & $1(<1)$ & 0 & $1(1)$ & 1 \\
\hline Not recorded & $1(<1)$ & 0 & $1(1)$ & 1 \\
\hline \multicolumn{5}{|l|}{ Stone characteristics } \\
\hline $\begin{array}{l}\text { Sum max diameter all stones }(\mathrm{mm}) \\
\text { mean (range) }\end{array}$ & $24(7-80)$ & $18(7-34)$ & $25(8-80)$ & $<0.001$ \\
\hline Sum max stone diameter $>40 \mathrm{~mm}$ & $15(13)$ & 0 & $15(16)$ & $<0.001$ \\
\hline Multiple stones & 57 & $13(50)$ & $44(48)$ & 1 \\
\hline Bilateral & $8(7)$ & 0 & $8(9)$ & 0.10 \\
\hline Complete staghorn & 5 & 0 & $5(6)$ & 0.32 \\
\hline \multicolumn{5}{|l|}{ Surgical factors } \\
\hline Access site - upper pole & $54(45)$ & $11(42)$ & $43(46)$ & 0.82 \\
\hline Access site - interpolar & $9(8)$ & $2(8)$ & $7(8)$ & 1 \\
\hline Access site - lower pole & $56(47)$ & $13(50)$ & $43(46)$ & 0.82 \\
\hline Multiple dilation tracts & $4(3)$ & 0 & $4(4)$ & 0.32 \\
\hline Transplant kidney & $1(<1)$ & 0 & $1(1)$ & 1 \\
\hline Solitary kidney & $2(2)$ & 0 & $2(2)$ & 1 \\
\hline Existing nephrostomy tube & $5(4)$ & 0 & $5(5)$ & 0.32 \\
\hline Existing stent & $1(<1)$ & 0 & $1(1)$ & 1 \\
\hline Operative time (min), mean (range) & $104(32-210)$ & $92(40-156)$ & $107(32-210)$ & 0.09 \\
\hline \multicolumn{5}{|l|}{ Exit strategy } \\
\hline Tubeless (stent only) & $116(98)$ & $25(96)$ & $91(99)$ & 0.47 \\
\hline Totally tubeless & $2(2)$ & $1(4)$ & $1(1)$ & 0.47 \\
\hline Nephrostomy tube & $0(0)$ & 0 & 0 & - \\
\hline \multicolumn{5}{|l|}{ Postoperative imaging } \\
\hline KUB & $96 / 114(84)$ & $22 / 25(85)$ & $74 / 89(83)$ & 0.76 \\
\hline CT & $11 / 114(10)$ & $1 / 25(4)$ & $10 / 89(11)$ & 0.45 \\
\hline US & $7 / 114(6)$ & $2 / 25(8)$ & $5 / 89(6)$ & 0.65 \\
\hline
\end{tabular}


ASA: American Society of Anesthesiologists; BMI: body mass index; CT: computed tomography; KUB: kidney, ureter, bladder radiography; PCNL: percutaneous nephrolithotomy; US: ultrasound. 


\begin{tabular}{|c|c|c|c|c|}
\hline Outcomes & $\begin{array}{c}\text { Overall, } \\
\text { n }(\%) \\
\mathbf{N}=118\end{array}$ & $\begin{array}{c}\text { Standard } \\
\text { criteria, } \\
\text { n }(\%) \\
\mathrm{N}=\mathbf{2 6} \\
\end{array}$ & $\begin{array}{c}\text { Extended } \\
\text { criteria, } \\
\text { n }(\%) \\
\mathbf{N}=92 \\
\end{array}$ & $\mathbf{P}$ \\
\hline Stone-free $(<3 \mathrm{~mm})$ & 95/114 (83) & $21 / 25(84)$ & $74 / 89(83)$ & 1 \\
\hline $\begin{array}{l}\text { If not stone-free, fragment size }(\mathrm{mm}) \text {, } \\
\text { average (range) }\end{array}$ & $4.9(3-11)$ & $5(3-8)$ & $4.7(3-11)$ & 0.8 \\
\hline Hemoglobin decrease $>20 \mathrm{~g} / \mathrm{L}$ & $5 / 106(5)$ & $3 / 21(14)$ & $2 / 85(2)$ & 0.052 \\
\hline Transfusions & 0 & 0 & 0 & - \\
\hline \multicolumn{5}{|l|}{$\begin{array}{l}\text { Clavien-Dindo grade (within } 6 \text { weeks of } \\
\text { surgery) }\end{array}$} \\
\hline Any & $20(17)$ & $3(12)$ & $17(18)$ & 0.56 \\
\hline I & $17(14)$ & $2(8)$ & $15(16)$ & 0.15 \\
\hline II & $1(<1)$ & $1(4)$ & 0 & 0.27 \\
\hline IIIa & 0 & 0 & 0 & - \\
\hline IIIb & $1(<1)$ & 0 & $1(1)$ & 1 \\
\hline IVa & $1(<1)$ & 0 & $1(1)$ & 1 \\
\hline $\begin{array}{l}\text { Emergency department visit within } 6 \\
\text { weeks of surgery }\end{array}$ & & $3(12)$ & $17(18)$ & 0.56 \\
\hline Stent colic & $10(8)$ & $2(8)$ & $8(9)$ & 0.73 \\
\hline Hematuria & $1(<1)$ & 0 & $1(1)$ & 1 \\
\hline Urinary retention & $4(3)$ & 0 & $4(4)$ & 0.56 \\
\hline Urinary tract infection & $1(<1)$ & 0 & $1(1)$ & 1 \\
\hline Cellulitis & $1(<1)$ & 0 & $1(1)$ & 1 \\
\hline Pyelonephritis & $1(<1)$ & $1(4)$ & 0 & 0.27 \\
\hline Pleural effusion & $1(<1)$ & 0 & $1(1)$ & 1 \\
\hline $\begin{array}{l}\text { Non-ST elevation myocardial } \\
\text { infarction }\end{array}$ & $1(<1)$ & 0 & $1(1)$ & 1 \\
\hline Readmission within 6 weeks of surgery & $6(5)$ & $1(4)$ & $5(5)$ & 1 \\
\hline Stent colic & $1(<1)$ & 0 & $1(1)$ & 1 \\
\hline Hematuria & $1(<1)$ & 0 & $1(1)$ & 1 \\
\hline Urinary retention & $1(<1)$ & 0 & $1(1)$ & 1 \\
\hline Pyelonephritis & $1(<1)$ & $1(4)$ & 0 & 0.27 \\
\hline Pleural effusion & $1(<1)$ & 0 & $1(1)$ & 1 \\
\hline $\begin{array}{l}\text { Non-ST elevation myocardial } \\
\text { infarction }\end{array}$ & $1(<1)$ & 0 & $1(1)$ & 1 \\
\hline
\end{tabular}

ASA: American Society of Anesthesiologists; BMI: body mass index; PCNL: percutaneous nephrolithotomy. 


\begin{tabular}{|c|c|c|}
\hline \multicolumn{3}{|c|}{ Table 3. Univariate analysis for complications after ambulatory PCNL ( $N=11$} \\
\hline Criteria & OR $(95 \%$ CI $)$ & $\mathbf{P}$ \\
\hline Age $>75$ & $0.98(0.11-8.86)$ & 0.99 \\
\hline $\mathrm{ASA}>2$ & $1.54(0.58-4.04)$ & 0.39 \\
\hline $\mathrm{BMI}>30$ & $1.30(0.48-3.51)$ & 0.60 \\
\hline Stone burden $>4 \mathrm{~cm}$ & $0.73(0.15-3.50)$ & 0.69 \\
\hline Transplant/solitary kidney & $2.53(0.22-29.29)$ & 0.46 \\
\hline Existing nephrostomy tube/stent & $2.61(0.44-15.34)$ & 0.29 \\
\hline 1 risk factor & $1.65(0.63-4.34)$ & 0.31 \\
\hline 2 risk factors & $1.18(0.41-3.41)$ & 0.75 \\
\hline$>2$ risk factors & $0.52(0.06-4.36)$ & 0.55 \\
\hline
\end{tabular}

ASA: American Society of Anesthesiologists; BMI: body mass index; CI: confidence interval; OR: odds ratio; PCNL: percutaneous nephrolithotomy.

\begin{tabular}{|c|c|c|c|c|}
\hline \multicolumn{5}{|c|}{$\begin{array}{l}\text { Table 4. Univariate and multivariable analysis for residual stones }>3 \mathbf{~ m m} \text { after } \\
\text { ambulatory PCNL (N=114) }\end{array}$} \\
\hline Criteria & $\begin{array}{c}\text { OR }(95 \% \mathrm{CI}) \\
\text { Univariate }\end{array}$ & $\mathbf{P}$ & $\begin{array}{l}\text { OR (95\% CI) } \\
\text { Multivariable }\end{array}$ & $\mathbf{P}$ \\
\hline Age $>75$ & $0.94(0.10-8.58)$ & 0.96 & & \\
\hline $\mathrm{ASA}>2$ & $0.91(0.33-2.47)$ & 0.85 & & \\
\hline $\mathrm{BMI}>30$ & $0.63(0.21-1.89)$ & 0.41 & & \\
\hline $\begin{array}{l}\text { Stone burden }>4 \\
\mathrm{~cm}\end{array}$ & $6.92(2.06-23.19)$ & 0.002 & $5.83(1.35-25.16)$ & 0.018 \\
\hline Bilateral stones & $4.03(0.82-19.75)$ & 0.09 & $0.82(0.09-7.51)$ & 0.86 \\
\hline Complete staghorn & $10.47(0.90-122.05)$ & 0.06 & $11.09(0.83-148.52)$ & 0.069 \\
\hline Multiple tracts & $16.69(1.63-170.64)$ & 0.02 & $13.10(1.11-154.72)$ & 0.041 \\
\hline 1 risk factor & $1.09(0.40-2.97)$ & 0.87 & & \\
\hline 2 risk factors & $0.98(0.32-3.02)$ & 0.98 & & \\
\hline$>2$ risk factors & $1.65(0.31-8.86)$ & 0.56 & & \\
\hline
\end{tabular}

$\mathrm{N}=114$ due to missing imaging in 4 patients. Multivariable analysis performed including variables with $\mathrm{p}<0.2$. ASA: American Society of Anesthesiologists; BMI: body mass index; CI: confidence interval; OR: odds ratio; PCNL: percutaneous nephrolithotomy. 


\begin{tabular}{|l|l|}
\hline $\begin{array}{l}\text { Supplementary Table 1. Occurrence of extended criteria } \\
\text { characteristics (N=92) }\end{array}$ \\
\hline Criteria & $\mathbf{N}(\boldsymbol{\%})$ \\
\hline Age $>75$ & $6(7)$ \\
\hline ASA $>2$ & $51(55)$ \\
\hline BMI $>30$ & $50(54)$ \\
\hline Stone burden >4 cm & $15(16)$ \\
\hline Bilateral stones & $8(9)$ \\
\hline Complete staghorn & $5(5)$ \\
\hline Multiple tracts & $4(4)$ \\
\hline 1 risk factor & $50(54)$ \\
\hline 2 risk factors & $32(35)$ \\
\hline$>2$ risk factors & $10(11)$ \\
\hline
\end{tabular}

ASA: American Society of Anesthesiologists; BMI: body mass index. 